\title{
Cellular uptake mechanism and intracellular fate of hydrophobically modified pullulan nanoparticles
}

This article was published in the following Dove Press journal:

International Journal of Nanomedicine

7 May 2013

Number of times this article has been viewed

Liqin Jiang'

Xuemin $\mathrm{Li}^{\prime}$

Lingrong Liu'

Qiqing Zhang ${ }^{1,2}$

'Institute of Biomedical Engineering, Chinese Academy of Medical Sciences and Peking Union Medical College, Tianjin, People's Republic of China; ${ }^{2}$ Research Center of Biomedical Engineering, Xiamen University, Xiamen, People's Republic of China

Correspondence: Qiqing Zhang Institute of Biomedical Engineering, Chinese Academy of Medical Science \& Peking Union Medical College, Baidi Road 236, Tianjin 300192, People's Republic of China

Tel +862287892052

Fax +86 2287892052

Email bmezqq@163.com
Abstract: The cellular uptake mechanism and intracellular fate of self-assembled nanoparticles (NPs) of cholesterol-modified pullulan (CHSP) by human hepatocellular carcinoma (HepG2) cells were investigated. Covalent conjugation with fluorescein isothiocyanate (FITC) yielded stably labeled CHSP (FITC-CHSP), which was successfully formulated into NPs (mean particle size $63.0 \pm 1.9 \mathrm{~nm}$ ) by dialysis. A cytotoxicity assay clearly indicated that the CHSP NPs did not show significant toxicity in HepG2 cells. The effects of NP concentration, incubation time, and temperature on the cellular uptake of the NPs were systematically evaluated by fluorometry, and the results suggested that cellular uptake of the NPs was concentration-, time-, and temperature-dependent. In vitro experiments with endocytic inhibitors revealed that clathrin-mediated endocytosis and macropinocytosis were involved in the internalization of CHSP NPs. The intracellular trafficking study demonstrated that CHSP NPs were entrapped in the lysosomes at 1 hour after incubation; colocalization of NPs with either the Golgi apparatus or the endoplasmic reticula was not observed during the entire course of the study. These results suggested that the CHSP NPs may serve as a versatile carrier for intracellular delivery of therapeutic agents.

Keywords: cholesterol-modified pullulan, self-assembled nanoparticles, FITC, endocytosis, intracellular trafficking

\section{Introduction}

Pullulan, a water-soluble and linear polysaccharide with maltotriose repeating units joined by $\alpha$-1,6-linkages, has been used in many research fields, including food, pharmaceutical, and biomedical sciences, due to its biocompatible and biodegradable nature. ${ }^{1-4}$ Hydrophobically modified pullulan can self-assemble into nanoparticles (NPs) in aqueous media by means of inter- or intramolecular interactions between its hydrophobic moieties, to minimize interfacial free energy. The self-assembled NPs, consisting of a hydrophobic core and hydrophilic shell, have been recognized as a promising drug carrier, since their hydrophobic domain can encapsulate various hydrophobic drugs. ${ }^{5}$

In recent years, many studies have been carried out to investigate the synthesis and the application of pullulan-based self-assembled NPs as drug delivery systems, ${ }^{6-12}$ among which, cholesterol-bearing pullulan (CHP), synthesized by the reaction between cholesteryl N-(6-isocynatohexyl)carbamate and pullulan using 1,6-hexyl diisocyanate linkages, has attracted much attention. CHP can form stable hydrogel NPs by self-assembly, ${ }^{13,14}$ and can then form complexes with various drugs and proteins by hydrophobic interaction and release them upon exposure to specific proteins 
or cyclodextrin. ${ }^{15-18}$ The hydrogel NPs can also be used as a drug carrier system in medicine ${ }^{17,19,20}$ as well as artificial molecular chaperones in biotechnology. ${ }^{21-23}$ On the basis of these, our group developed a new method to synthesize cholesterol-modified pullulan (CHSP) by directly grafting proper cholesterol residues onto pullulan using succinyl linkages, thus providing a new strategy for the effective and safe synthesis of CHSP. In vitro studies indicated that CHSP self-assembled NPs showed potential as a sustained-release carrier of mitoxantrone. ${ }^{24}$

To improve the therapeutic potential of the NP-based carriers for intracellular delivery, it is important to understand the cellular uptake mechanism and the intracellular fate of NPs. To date, a few reports have discussed the internalization of self-assembled NPs of hydrophobically modified chitosan into the cells by endocytic pathways. ${ }^{25,26}$ However, the cellular uptake mechanism and intracellular fate of self-assembled NPs of hydrophobically modified pullulan remains to be understood. Fluorescently labeled NPs can provide a rapid, simple, and sensitive means to quantify cell-associated NPs by fluorometry. ${ }^{27-29}$ In the present study, fluorescein isothiocyanate (FITC) was covalently bound to the pullulan, and self-assembled NPs of FITC-labeled CHSP (FITC-CHSP) were prepared by dialysis. Human hepatocellular carcinoma cells (HepG2 cells) were chosen as an in vitro model, and the cellular uptake of the NPs was quantified by fluorometry, to investigate the effect of NP concentration, incubation time, and temperature, on the uptake. Meanwhile, several selective endocytosis inhibitors, chlorpromazine, filipin, and amiloride, were selected to study the potential endocytosis mechanism. Furthermore, the intracellular fate of CHSP NPs was investigated by confocal laser scanning microscopy (CLSM).

\section{Materials and methods}

\section{Preparation and characterization of FITC-CHSP}

The synthetic route of CHSP was described in our previous report. ${ }^{24}$ FITC-CHSP was prepared by the method of Kaneo et al. ${ }^{30}$ Briefly, CHSP ( $1 \mathrm{~g}$ ) was dissolved in dimethyl sulfoxide (DMSO; Sigma-Aldrich, St Louis, MO, USA) (10 mL) containing a few drops of pyridine. FITC (Sigma-Aldrich; $100 \mathrm{mg}$ ) was added, followed by dibutyltin dilaurate (SigmaAldrich; $20 \mathrm{mg}$ ), and the mixture was heated for 2 hours at $95^{\circ} \mathrm{C}$. After several precipitations in ethanol to remove free dye, the FITC-CHSP fraction was filtered off and dried in vacuo at $80^{\circ} \mathrm{C}$. The synthesized FITC-CHSP was further dialyzed, in the dark, against deionized water and replaced on a daily basis until no fluorescence was detected in the supernatant.
The resultant FITC-CHSP were lyophilized in a freeze dryer and used to prepare the FITC-CHSP NPs as described above. The chemical structure of the FITC-CHSP conjugates obtained was characterized by Fourier transform infrared spectroscopy (FT-IR) (Nicolet ${ }^{\mathrm{TM}}$ Nexus 470-ESP; Thermo Fisher Scientific, Waltham, MA, USA).

Labeling efficiency was calculated as the percent weight of conjugated FITC to the weight of FITC-CHSP. To determine the labeling efficiency, the fluorescence intensity of a solution of FITC-CHSP, dissolved in DMSO and appropriately diluted with phosphate-buffered saline (PBS) $(\mathrm{pH}$ 7.4), was measured in the fluorometer (LS-50B, PerkinElmer Inc, Waltham, MA, USA) ( $\lambda \operatorname{exc} 490 \mathrm{~nm}, \lambda$ emi $520 \mathrm{~nm})$. The fluorometer was calibrated with different concentration FITC standard solutions prepared by diluting $100 \mu \mathrm{g} / \mathrm{mL}$ DMSO solutions of FITC with PBS (pH 7.4).

\section{Preparation and characterization of FITC-CHSP self-assembled NPs}

FITC-CHSP self-assembled NPs were prepared by a dialysis method. In brief, FITC-CHSP was dissolved in anhydrous DMSO, and then the solution was transferred into a preswollen dialysis membrane (molecular weight cutoff [MWCO] 12-14 kDa) (SpectraPor ${ }^{\circledR}$ RC; Spectrum Laboratories Inc, Rancho Dominguez, CA, USA) and dialyzed against deionized water for 24 hours at room temperature (deionized water was exchanged at 3-hour intervals). The dialysate was filtered with a $0.45 \mu \mathrm{m}$ filter to remove the precipitated material. The morphology of the FITC-CHSP self-assembled NPs was observed using a transmission electron microscope (TEM) (JEM-100CXII, JEOL Ltd, Tokyo, Japan). The particle size and size distribution of the NPs were measured by dynamic laser light scattering (DLS) (Zetasizer Nano-ZS; Malvern Instruments Ltd, Worcestershire, UK).

\section{In vitro release of FITC from NPs}

The in vitro release profiles of FITC from the NPs were evaluated as follows: $2 \mathrm{~mL}$ of the FITC-CHSP NP suspension was transferred to a dialysis tube (MWCO 12-14 kDa) (D-Tube ${ }^{\mathrm{TM}}$ Dialyzer Maxi; EMD Millipore, Billerica, MA, USA), and the dialysis tube was immersed in $10 \mathrm{~mL}$ of PBS ( $\mathrm{pH} 7.4$ ). The tube was stirred at $100 \mathrm{rpm}$ at $37^{\circ} \mathrm{C}$. At predetermined time intervals, the release medium was collected and the fresh release medium was then added. Each sample was then subjected to centrifugation at $10,000 \times \mathrm{g}$ for 8 minutes $(5810 \mathrm{R}$, Ependorff AG, Hamburg, Germany), and the concentration of FITC in the supernatant was measured in the fluorometer ( $\lambda$ exc $490 \mathrm{~nm}, \lambda$ emi $520 \mathrm{~nm}$ ). Unlabeled CHSP NPs were 
also prepared by dialysis. The fluorometer was calibrated with standard solutions containing 0.002 to $0.1 \mu \mathrm{g} / \mathrm{mL}$ of FITC dissolved in the supernatant generated from unlabeled CHSP NP suspension that underwent the same procedures as above. Analyses were performed in triplicate. The cumulative release percentage $(\mathrm{Q} \%)$ was calculated by the following equation:

$$
\mathrm{Q} \%=\frac{\mathrm{C}_{\mathrm{n}} \times \mathrm{V}+\mathrm{V}_{\mathrm{i}} \sum_{\mathrm{i}=0}^{\mathrm{n}-1} \mathrm{C}_{\mathrm{i}}}{\mathrm{W}_{\text {FITC-CHSP NPs }} \times \mathrm{LE} \%} \times 100 \%,
$$

where $\mathrm{C}_{\mathrm{n}}$ is the sample concentration at $\mathrm{T}_{\mathrm{n}}, \mathrm{V}$ is the total volume of release medium, $\mathrm{V}_{\mathrm{i}}$ is the sample volume at $\mathrm{T}_{\mathrm{i}}, \mathrm{C}_{\mathrm{i}}$ is the sample concentration at $\mathrm{T}_{\mathrm{i}}$ (both $\mathrm{V}_{0}$ and $\mathrm{C}_{0}$ were equal to zero), and $\mathrm{LE} \%$ is labeling efficiency.

\section{Cell culture}

HepG2 cells were cultured in Dulbecco's modified Eagle's medium (DMEM; Thermo Fisher Scientific, Waltham, MA, USA) (25 mmol/L glucose) supplemented with 10\% fetal bovine serum (FBS; Sigma-Aldrich), $100 \mathrm{IU} / \mathrm{mL}$ penicillin (PAA Laboratories, Pasching, Austria), and $100 \mu \mathrm{g} / \mathrm{mL}$ streptomycin (PAA Laboratories), at $37^{\circ} \mathrm{C}$ using a humidified $5 \% \mathrm{CO}_{2}$ incubator.

\section{Cytotoxicity}

The cytotoxicity of CHSP NPs against HepG2 cells was evaluated by 3-(4,5-Dimethylthiazol-2-yl)-2,5-diphenyltetrazolium bromide (MTT) assay (Sigma-Aldrich). The principle of MTT assay is a reduction in metabolic activity, which is an early indication of cellular damage. ${ }^{31}$ The cells at logarithmic growth phase were seeded in a 96-well plate (Nunc ${ }^{\mathrm{TM}}$ Edge 96-Well Plate; Thermo Fisher Scientific) at $5.0 \times 10^{3}$ cells/well and grown for 24 hours. The culture medium was then replaced by $200 \mu \mathrm{L}$ of DMEM containing different concentrations of CHSP NPs, and the cells were further incubated for 24 hours and 72 hours, respectively. DMEM without NPs was used for control. Following incubation, the cells were washed three times with the culture medium. Then, $100 \mu \mathrm{L}$ of DMEM and $20 \mu \mathrm{L}$ of MTT solution ( $5 \mathrm{mg} / \mathrm{mL}$ in PBS) were added to each well. After incubation for another 4 hours, the MTT medium was removed, $150 \mu \mathrm{L}$ of DMSO was added, and then the mixture was shaken for 10 minutes at room temperature to dissolve the reacted dye. The absorbance was measured at $570 \mathrm{~nm}$ with a microplate reader (Varioskan Flash; Thermo Fisher Scientific). The percentage of cell viability (CV\%) was calculated as follows:

$$
\mathrm{CV} \%=\frac{\mathrm{OD}_{\text {treated }}}{\mathrm{OD}_{\text {control }}} \times 100 \% \text {, }
$$

where $\mathrm{OD}_{\text {treat }}$ and $\mathrm{OD}_{\text {control }}$ are the absorbances of the treated cells and the control cells.

\section{NP uptake by HepG2 cells}

The capacity for cellular internalization of FITC-CHSP NPs was visualized and quantified by CLSM and fluorometry, respectively. HepG2 cells were seeded at a density of $1.6 \times 10^{5}$ cells $/ \mathrm{cm}^{2}$ in glass bottom culture dishes $(\mathrm{P} 35 \mathrm{G}-$ 0-10-C, MatTek Corp., Ashland, MA, USA) and incubated. Subsequently, cells were rinsed twice with PBS (pH 7.4) and replenished with $1 \mathrm{~mL}$ serum-free medium containing the FITC-CHSP NPs for different time intervals $(0.5,1,2,3,4$, 5, 6 hours). After incubation, test samples were aspirated. Cells were then washed twice with prewarmed PBS before they were fixed in $4 \%$ paraformaldehyde. Finally, the fixed cells were counterstained with 4',6-diamidino-2-phenylindole (DAPI) (Sigma-Aldrich, St Louis, MO, USA) to visualize nuclei and were examined under a CLSM (TCS SL; Leica Microsystems, Wetzlar, Germany).

To quantify the cellular uptake of NPs, cells were plated in 12-well plates (Costar ${ }^{\circledR}$, Corning Inc, Corning, NY, USA) at a density of $1.6 \times 10^{5}$ cells $/ \mathrm{cm}^{2}$ and incubated with a FITC-CHSP NP suspension in serum-free medium, washed three times with PBS ( $\mathrm{pH} 7.4$ ) and then lysed by incubating them with $1 \mathrm{~mL}$ of $0.5 \%$ Triton $^{\mathrm{TM}} \mathrm{X}-100$ (Sigma-Aldrich) in $0.2 \mathrm{~N} \mathrm{NaOH}$. Cell-associated FITC-CHSP NPs were quantified by analyzing the cell lysate in a microplate reader ( $\lambda$ exc $490 \mathrm{~nm}, \lambda$ emi $520 \mathrm{~nm}$ ) calibrated with standard solutions containing 3.5 to $60.0 \mu \mathrm{g} / \mathrm{mL}$ of FITC-CHSP NPs in a cell lysate solution $\left(2 \times 10^{5}\right.$ untreated HepG2 cells dissolved in $1 \mathrm{~mL}$ of the Triton $\mathrm{X}-100$ solution). Uptake was expressed as the amount $(\mu \mathrm{g})$ of FITC-CHSP NPs associated with unit weight (mg) of cellular protein. The protein content of the cell lysate was measured using the Pierce BCA Protein Assay kit (Thermo Fisher Scientific).

Initially, the concentration- and time-dependent cellular uptake of NPs was determined. To study the concentrationdependent NP uptake, cells were incubated with different concentrations of NP suspension $(0.05-2 \mathrm{mg} / \mathrm{mL})$ for 4 hours. To study the time-dependent NP uptake, cells were incubated with a suspension of NPs $(0.15 \mathrm{mg} / \mathrm{mL})$ for different time periods.

\section{Endocytosis inhibition}

The effect of temperature block was studied by preincubating the cells at $4^{\circ} \mathrm{C}$ for 1 hour, followed by treatment with the FITC-CHSP NPs for 2 hours at $4^{\circ} \mathrm{C} .{ }^{32}$ To study the effect of various inhibitors on the uptake of NPs, cells were 
preincubated with the following inhibitors individually, at concentrations which were nontoxic to the cells: $7 \mu \mathrm{g} / \mathrm{mL}$ of chlorpromazine (Sigma-Aldrich), ${ }^{33,34}$ to inhibit the clathrinmediated endocytosis, $1 \mu \mathrm{g} / \mathrm{mL}$ of filipin (Sigma-Aldrich), ${ }^{32}$ to inhibit caveolae-mediated endocytosis, or $50 \mu \mathrm{M}$ amiloride (Sigma-Aldrich), ${ }^{26}$ to inhibit macropinocytosis. Following the preincubation for 1 hour at $37^{\circ} \mathrm{C}$, the inhibitor solutions were removed, and the freshly prepared FITC-CHSP NPs, in medium containing inhibitors at the same concentrations, were added and further incubated for 2 hours. Subsequently, the cells were washed three times with PBS and analyzed according to the methods mentioned above. The group in the presence of FITC-CHSP NPs but without inhibitor treatment was used as control, and their uptake was expressed as $100 \%$.

\section{Investigation of intracellular fate of CHSP NPs}

To study the intracellular fate of CHSP NPs, immunofluorescence staining was used to identify the Golgi apparatus, endoplasmic reticula (ER), and lysosomes. The antibodies used in the study were mouse monoclonal Anti-Giantin antibody (9B6) - Golgi Marker (ab37266; Abcam, Cambridge, UK), mouse monoclonal Anti-Calnexin ER membrane marker antibody (AF18) (ab31290; Abcam), mouse monoclonal Anti-lysosomal-associated membrane protein [LAMP]2 antibody (H4B4) - Lysosome marker (ab25631; Abcam), and goat Anti-Mouse IgG (H+L), CFTM 647 antibody (SAB4600182; Sigma-Aldrich). Briefly, cells were treated with the FTICCHSP NPs in the serum-free medium. After incubation, at predetermined time points, cells were washed twice with the prewarmed PBS, fixed in 4\% paraformaldehyde, and then washed an additional three times with PBS. Cells were permeabilized (with PBS containing 0.3\% Triton X-100 [v/v], 0.1\% bovine serum albumin (BSA; Sigma-Aldrich) [w/v]) for 15 minutes at room temperature. The samples were blocked with dilution buffer (PBS containing 10\% goat serum (v/v), $0.3 \mathrm{M}$ glycine) for 1 hour and then incubated with the primary antibody (1:200 for Golgi marker; 1:1000 for ER membrane marker and Lysosome marker) for 1 hour at room temperature. Cells were washed three times with permeabilization buffer and then incubated with the secondary antibody (1:1000, goat antimouse $\operatorname{IgG}(\mathrm{H}+\mathrm{L}), \mathrm{CF}$ 647A antibody) for 45 minutes at room temperature. After the secondary antibody incubation, cells were washed twice with the permeabilization buffer, counterstained to visualize nuclei with DAPI, and examined by CLSM (LSM 710; Carl Zeiss Meditec Inc, Jena, Germany).

\section{Statistical analysis}

All data were presented as mean \pm standard deviation. Statistical analysis was conducted by using the Student's $t$-test, with $P<0.05$ as the significant difference.

\section{Results and discussion Characterization of FITC-CHSP}

In this study, CHSP (with the substitution of 5.40 cholesterol residues per 100 glucose units in the pullulan), was used to prepare FITC-CHSP. Figure 1 shows the FT-IR spectra of FITC, CHSP, and FITC-CHSP. The spectra of FITC (Figure 1A) displayed a hydroxy characteristic peak at $3423 \mathrm{~cm}^{-1}$ and a strong isothiocyanate characteristic peak at $2015 \mathrm{~cm}^{-1}$ as well as a characteristic peak assigned to the carbonyl group of lactone, at around $1727 \mathrm{~cm}^{-1}$. Furthermore, the absorption peaks at 1594,1535 , and $1458 \mathrm{~cm}^{-1}$ were characteristics of benzene ring stretching vibration. For FITCCHSP (Figure 1C), the peak at $2930 \mathrm{~cm}^{-1}$ was attributed to the stretching vibration of methyl and methylene groups, and the characteristic peak observed at $1733 \mathrm{~cm}^{-1}$ was assigned to the carbonyl group. Compared with FITC, the isothiocyanate

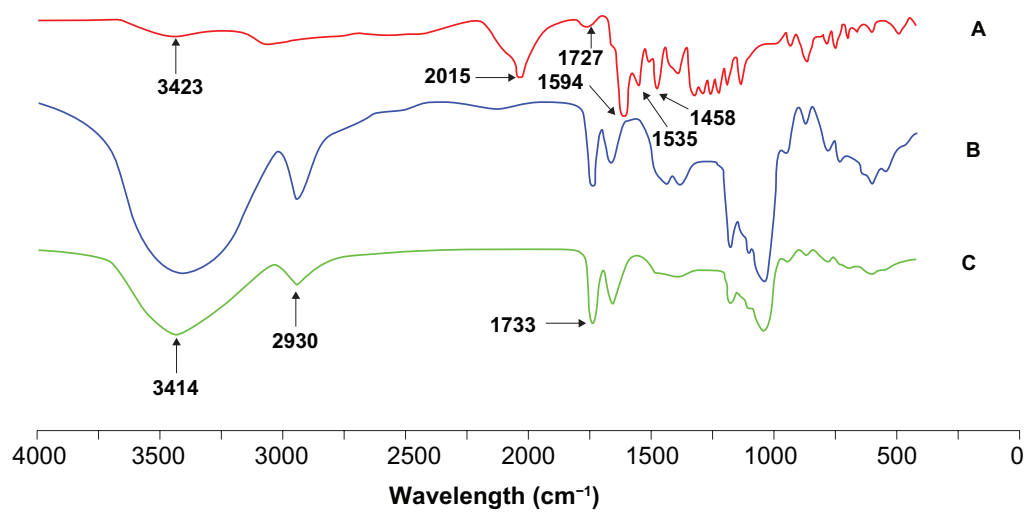

Figure I FT-IR spectra of (A) FITC (B) CHSP and (C) FITC-CHSP.

Abbreviations: CHSP, cholesterol-modified pullulan; FITC, fluorescein isothiocyanate; FT-IR, Fourier transform infrared spectroscopy. 
A

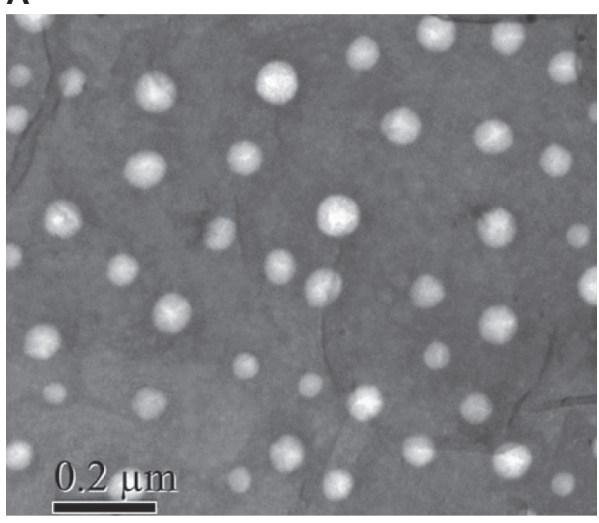

\section{B}

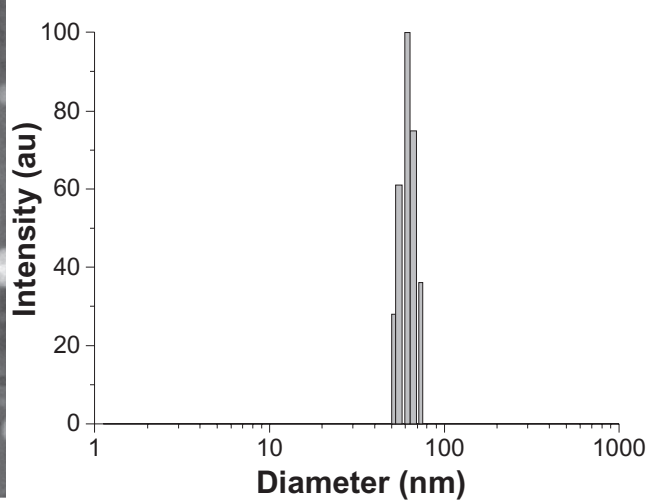

Figure 2 (A) Morphological characteristics and (B) size distribution of FITC-CHSP NPs, as determined by TEM and DLS, respectively.

Abbreviations: DLS, dynamic light scattering; FITC-CHSP, fluorescein isothiocyanate-labeled cholesterol-modified pullulan; NP, nanoparticle; TEM, transmission election microscopy.

characteristic peak at $2015 \mathrm{~cm}^{-1}$ disappeared in the spectra of FITC-CHSP, which suggested the isothiocyanate group was involved in the addition reaction with the hydroxyl group. The labeling efficiency, calculated as the percent weight of conjugated FITC to weight of FITC-CHSP, was 3.0\%.

\section{Characterization of FITC-CHSP self-assembled NPs}

The amphiphilic FITC-CHSP conjugates can form selfassembled NPs in aqueous solution, due to hydrophobic inter- and intramolecular interactions. Figure 2A shows the morphological characteristics of the FITC-CHSP NPs. The NPs had an almost spherical shape and were well dispersed, without any aggregation. Since all the uptake study was performed in serum-free medium, we characterized NPs suspended in serum-free medium. Figure $2 \mathrm{~B}$ shows the particle

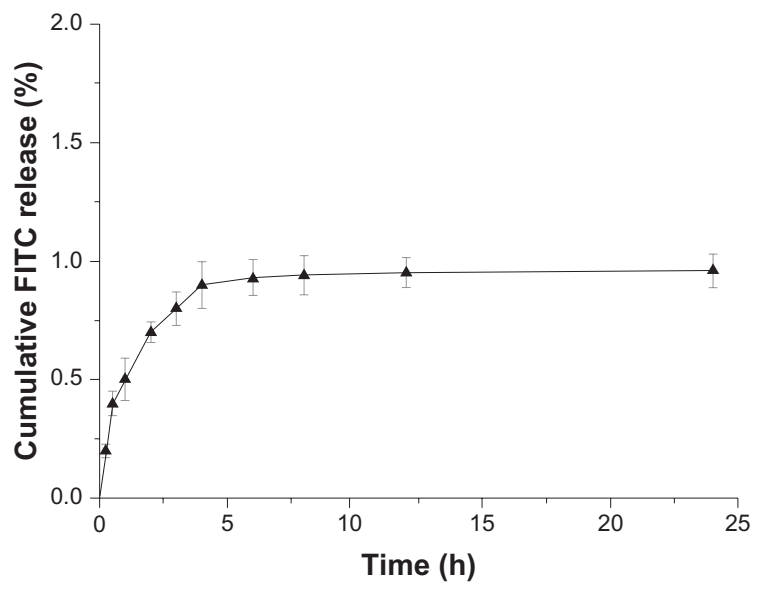

Figure 3 In vitro release profiles of FITC from FITC-CHSP NPs in PBS ( $\mathrm{pH} 7.4)$, at $37^{\circ} \mathrm{C}$.

Note: Data represents means \pm SD $(n=3)$.

Abbreviations: CHSP,cholesterol-modified pullulan;FITC,fluorescein isothiocyanate; NP, nanoparticle; PBS, phosphate-buffered saline; SD, standard deviation. size distribution of FITC-CHSP NPs, as measured by DLS The results indicate that the average diameter of NPs was $63.0 \pm 1.9 \mathrm{~nm}$, with a narrow size distribution. In addition, the mean diameter of NPs was not remarkably changed for 3 weeks, and no precipitates were found in the solution.

\section{In vitro release of FITC from NPs}

The use of fluorescent markers in NP visualization can lead to the misinterpretation of NP uptake data, due to the leaching or dissociation of fluorescent markers into the released medium $^{35}$ and hence, subsequently into the cells. Thus, the in vitro release study of FITC from FITC-CHSP NPs was conducted, and the results were shown in Figure 3. It can be found that less than $1 \%$ of the conjugated FITC was released over 24 hours, which was considered negligible in comparison with the NP uptake outcome of the HepG2 cells. It is thus

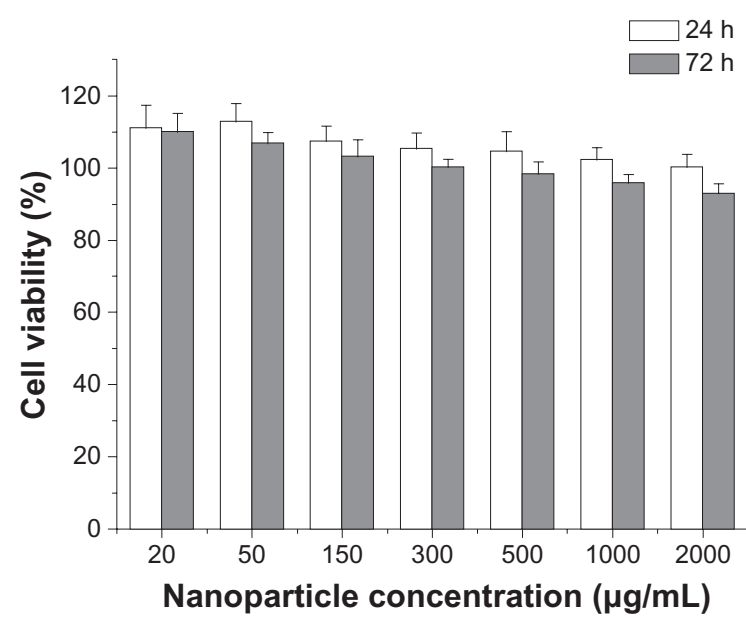

Figure 4 Cytotoxicity of CHSP NPs against HepG2 cells. Note: Data represents means $\pm S D(n=6)$.

Abbreviations: CHSP, cholesterol-modified pullulan; HepG2, hepatocellular carcinoma; NP, nanoparticle; SD, standard deviation. 


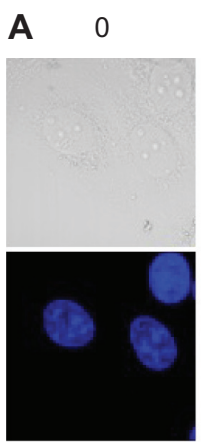

B 0
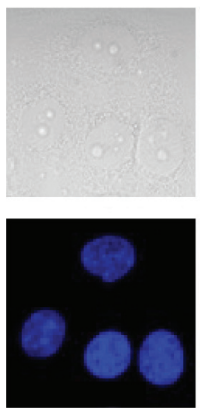

$0.05 \mathrm{mg} / \mathrm{mL}$

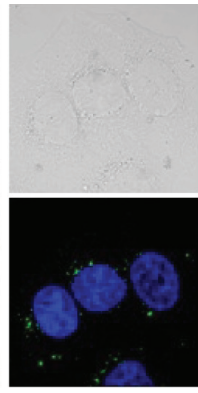

$30 \mathrm{~min}$
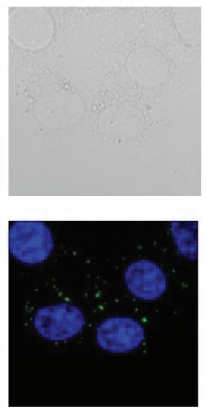

$0.15 \mathrm{mg} / \mathrm{mL}$

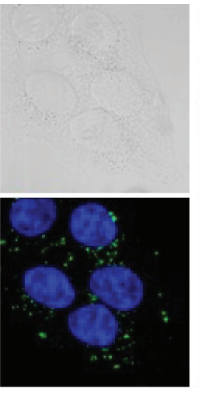

$1 \mathrm{~h}$
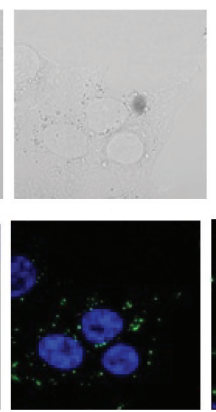

$0.3 \mathrm{mg} / \mathrm{mL}$

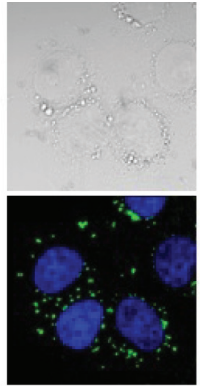

$2 \mathrm{~h}$
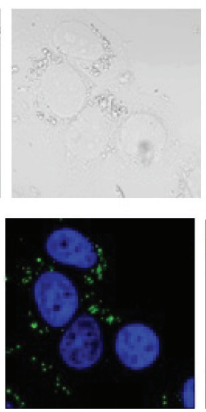

$0.5 \mathrm{mg} / \mathrm{mL}$

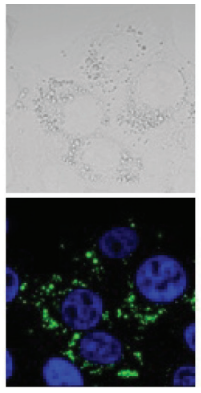

$4 \mathrm{~h}$
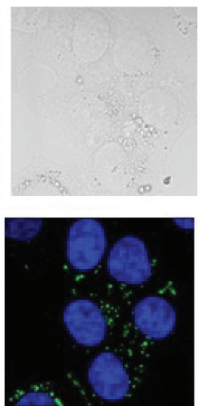

$2 \mathrm{mg} / \mathrm{mL}$

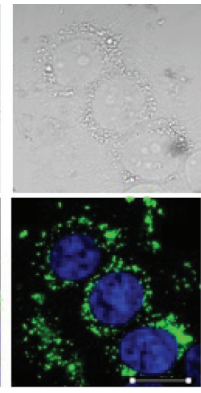

$6 \mathrm{~h}$
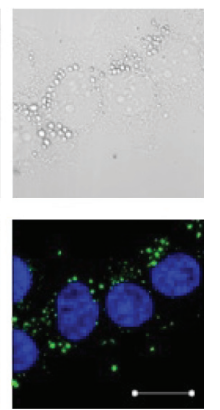

Figure 5 Cellular uptake profile of CHSP NPs as a function of $(\mathbf{A}) \mathrm{NP}$ concentration $\left(4\right.$ hours, $\left.37^{\circ} \mathrm{C}\right)$ and $(\mathbf{B})$ incubation time $\left(0.15 \mathrm{mg} / \mathrm{mL}, 37^{\circ} \mathrm{C}\right)$.

Notes: HepG2 cells were incubated with FITC-CHSP NPs under the condition indicated above each image, and the samples were prepared for CLSM investigation (scale bar, $20 \mu \mathrm{m})$.

Abbreviations: CHSP, cholesterol-modified pullulan; CLSM, confocal laser scanning microscopy; FITC, fluorescein isothiocyanate; HepG2, hepatocellular carcinoma; NP, nanoparticle.

reasonable to assume that most of the FITC was associated in the NPs and that the fluorescence measured from the uptake samples mainly reflects the cellular-associated fluorescent NPs but not the released fluorescence in the medium.

\section{Cytotoxicity}

To test the safety of CHSP NPs, we evaluated the cytotoxicity to HepG 2 proliferation over a range of concentrations $(20,50,150,300,500,1000$, and $2000 \mu \mathrm{g} / \mathrm{mL})$. As shown in Figure 4, after a 24-hour exposure to CHSP NPs at different concentrations, the cell viabilities of HepG2 cells were all over $100 \%$. Furthermore, CHSP NPs did not show significant toxicity at the concentrations applied in this study, at 72 hours. The cell viability of HepG 2 cells was up to $93.0 \%$, even at the highest concentration tested $(2000 \mu \mathrm{g} / \mathrm{mL})$ for 72 hours, which clearly indicated that CHSP NPs exhibited excellent cell viability in HepG2 cells.

\section{NP uptake by HepG2 cells}

CLSM images of cellular uptake of CHSP NPs as a function of NP concentration and incubation time were shown in Figure 5. Evidently, the degree of NP uptake was clearly concentration-dependent in the studied concentration range of $0.05 \mathrm{mg} / \mathrm{mL}$ to $2 \mathrm{mg} / \mathrm{mL}$ (Figure $5 \mathrm{~A}$ ). To investigate the effect of incubation time on NP uptake, CHSP NPs
$(0.15 \mathrm{mg} / \mathrm{mL})$ were added to the cultured HepG2 cells and incubated at $37^{\circ} \mathrm{C}$. At 30 minutes postincubation, the fluorescence signal from the CHSP NPs was detected primarily near the cellular membrane (Figure 5B). As the incubation time increased, the CHSP fluorescence became more intense inside the cells. However, there was no visible difference in fluorescence intensity beyond the 4-hour incubation.

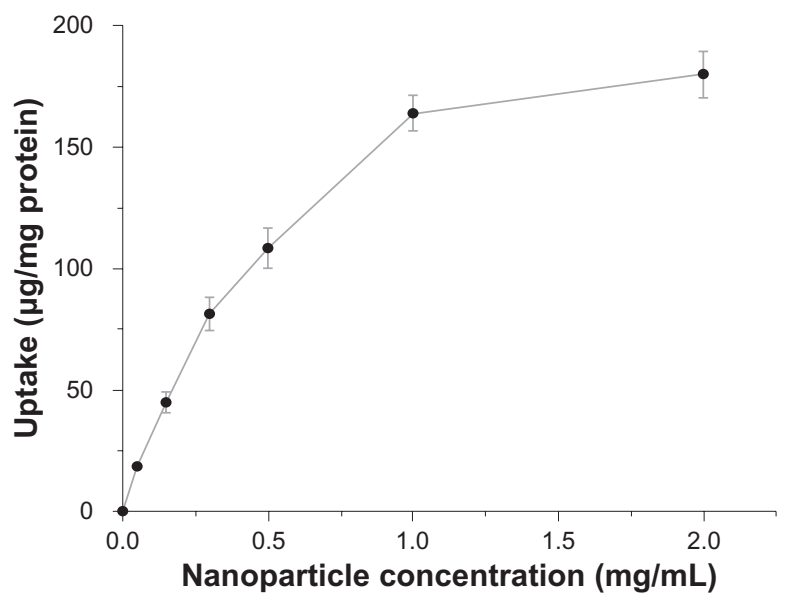

Figure 6 Effect of NP concentration on cellular uptake by HepG2 cells, at $37^{\circ} \mathrm{C}$. Notes: Various NP concentrations ranging from 0.05 to $2 \mathrm{mg} / \mathrm{mL}$ were applied to the HepG2 culture for 4 hours. Data represents mean \pm SD $(n=4)$.

Abbreviations: HepG2, hepatocellular carcinoma; NP, nanoparticle; SD, standard deviation. 


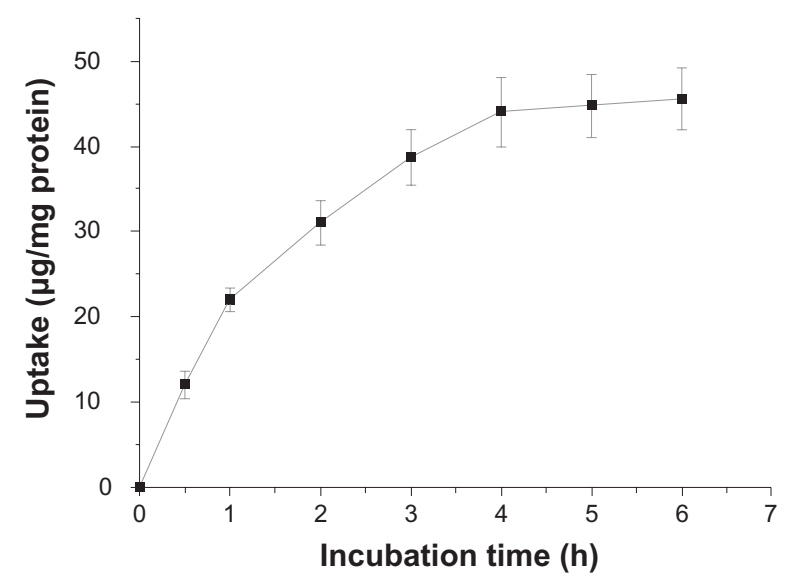

Figure 7 Effect of incubation time on NP uptake by HepG2 cells, at $37^{\circ} \mathrm{C}$. Notes: The concentration of NPs used was $0.15 \mathrm{mg} / \mathrm{mL}$. Data represents mean \pm $\mathrm{SD}(\mathrm{n}=4)$.

Abbreviations: HepG2, hepatocellular carcinoma; NP, nanoparticle; SD, standard deviation.

To quantify the cellular uptake of CHSP NPs, a series of standard solutions containing different amounts of FITC-CHSP NPs in a cell lysate solution $\left(2 \times 10^{5}\right.$ untreated HepG2 cells dissolved in $1 \mathrm{~mL}$ of the Triton X-100 solution) were tested to determine the linear relationship between the concentration of FITC-CHSP NPs and the corresponding fluorescence intensity. The results showed that fluorescence intensity (F) in the cell lysate solution varied linearly with concentration (C) of FITC-CHSP NPs in the range of 3.5 to $60.0 \mu \mathrm{g} / \mathrm{mL}$, obeying the following linear regression:

$$
\mathrm{F}=16.4 \mathrm{C}-21.0\left(\mathrm{R}^{2}=0.995\right) .
$$

As shown in Figure 6, the uptake of CHSP NPs by the HepG2 cells was concentration-dependent, and the uptake amount increased with increasing NP concentration from 0.05 to $2.0 \mathrm{mg} / \mathrm{mL}$, while the efficiency of uptake was reduced at higher doses. Figure 7 indicates that uptake of CHSP NPs by HepG2 cells at $37^{\circ} \mathrm{C}$ was also dependent on the incubation time and increased with the incubation time over a 4-hour period. However, the NP uptake had no significant further increase beyond 4 hours, since the curves showed a plateau effect after that time, which could be due to the limited saturation level.

\section{Endocytosis inhibition}

Temperature-dependent uptake studies were carried out with three different NP concentrations $(0.3,0.6$, and $0.9 \mathrm{mg} / \mathrm{mL})$ at both $37^{\circ} \mathrm{C}$ and $4^{\circ} \mathrm{C}$. There was a significant reduction $(P<0.05)$ in CHSP NP uptake by HepG 2 cells at $4^{\circ} \mathrm{C}$, with the uptake being reduced to $12 \%-15 \%$ of that at $37^{\circ} \mathrm{C}$ at equivalent dosing concentrations (Figure 8). These results suggest that an energy-dependent endocytic process could be responsible

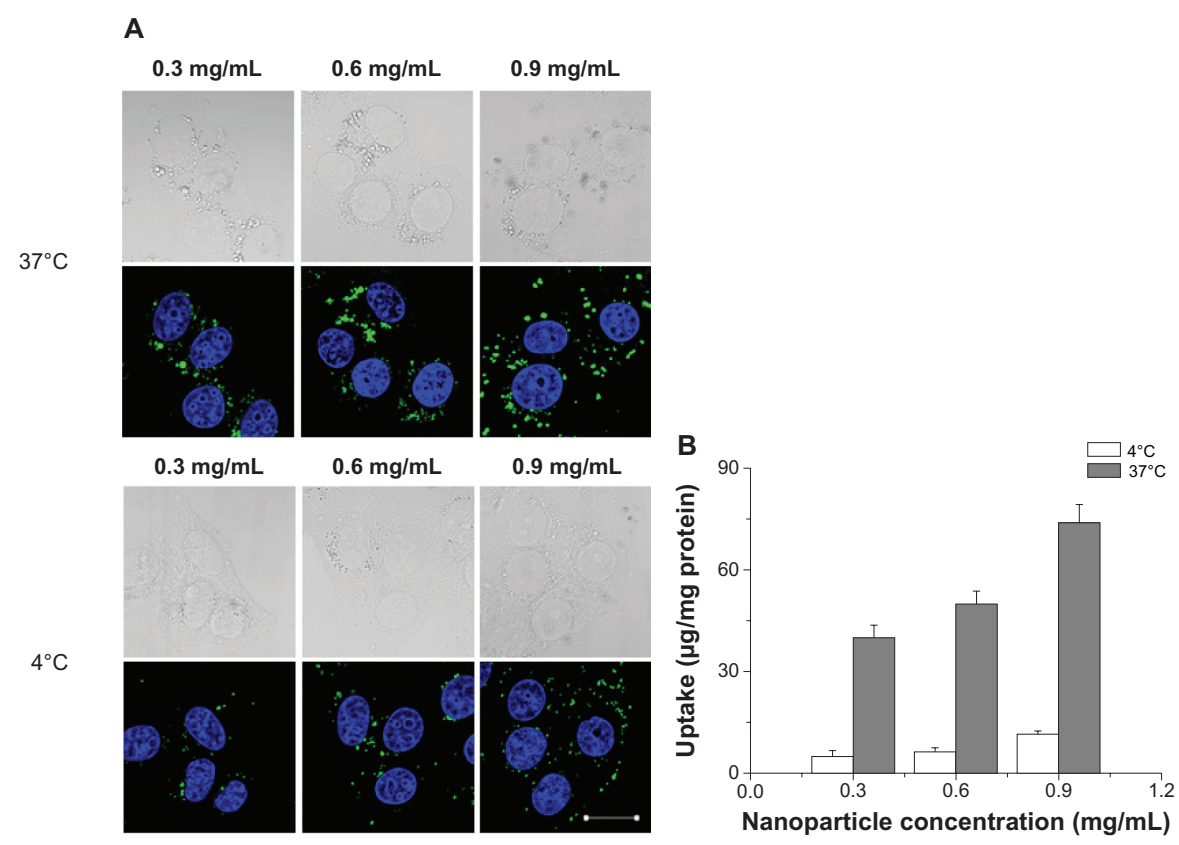

Figure 8 Effect of temperature on nanoparticle uptake by HepG2 cells. (A) Confocal images showing cellular uptake of CHSP NPs; (B) quantification of cellular uptake of CHSP NPs by fluorometry.

Notes: Two different temperatures $4^{\circ} \mathrm{C}$ and $37^{\circ} \mathrm{C}$ were used to evaluate the uptake of three different concentrations of NPs $(0.3,0.6$, and $0.9 \mathrm{mg} / \mathrm{mL})$. For $(\mathbf{A})$, the scale bar $=20 \mu \mathrm{m}$; Data in $(B)$ represent mean \pm SD $(n=4)$.

Abbreviations: CHSP, cholesterol-modified pullulan; HepG2, hepatocellular carcinoma; NP, nanoparticle; SD, standard deviation. 
for up to about $88 \%$ of NP uptake, while physical adhesion or diffusion probably describes the remaining uptake of NPs.

To elucidate their potential cellular uptake pathways, the interaction between CHSP NPs and cell membranes was investigated by treating the cells with different chemical inhibitors, of clathrin-mediated endocytosis, macropinocytosis, and caveolae-mediated endocytosis, and then analyzed by fluorometry. The inhibition of clathrin-mediated endocytosis was tested by using the cationic amphiphilic drug chlorpromazine, which causes clathrin to accumulate in late endosomes, thereby inhibiting coated pit endocytosis. ${ }^{36-39}$ Compared with the control, the cellular uptake of CHSP NPs was reduced by $\sim 55 \%$ $(P<0.05)$ (Figure 9), indicating that clathrin-mediated endocytosis may be involved. Caveolae, a specialized type of lipid rafts, are flask-shaped invaginations in the plasma membrane, enriched in proteins as well as cholesterol and sphingolipids. ${ }^{40,41}$ Filipin $(1 \mu \mathrm{g} / \mathrm{mL})$, an inhibitor of caveolae-mediated transport processes, ${ }^{42}$ did not affect NP uptake by the HepG2 cells $(P>0.05)$, which might be due to the absence of caveosomes in HepG2 cells, as reported in the literature. ${ }^{43}$ Alternatively, the cells treated with amiloride $(50 \mu \mathrm{M})$, a specific inhibitor of the $\mathrm{Na}^{+} / \mathrm{H}^{+}$exchange required for macropinocytosis, ${ }^{44}$ caused a significant decrease in NP uptake by $\sim 30 \%(P<0.05)$. Taken together, our inhibition studies suggest that clathrin-mediated endocytosis and macropinocytosis may be involved in the internalization of CHSP NPs.

\section{Investigation of intracellular fate of CHSP NPs}

Generally, NPs are taken up by cellular systems through endocytosis. ${ }^{45}$ Endocytosis, via clathrin-coated pits or

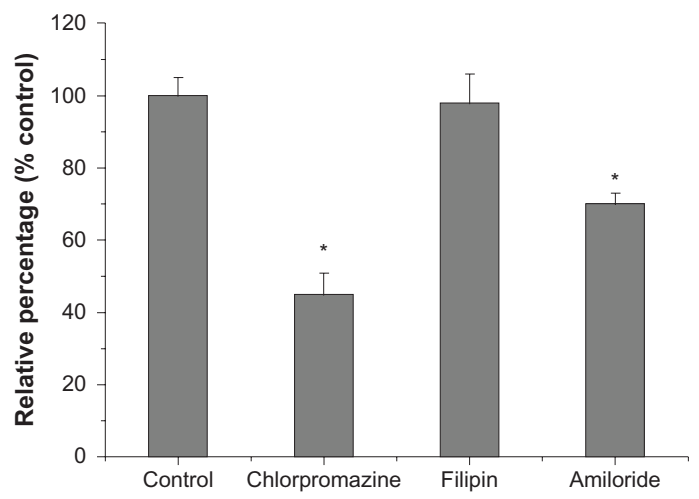

Figure 9 Effect of endocytic inhibitor on the internalization of FITC-CHSP NPs. Notes: HepG2 cells were pretreated with chlorpromazine $(7 \mu \mathrm{g} / \mathrm{mL})$, filipin $(\mathrm{I} \mu \mathrm{g} / \mathrm{mL})$, or amiloride $(50 \mu \mathrm{M})$, in serum-free medium for I hour, and then treated with FITC-CHSP NPs $\left(0.15 \mathrm{mg} / \mathrm{mL}, 2\right.$ hours, $\left.37^{\circ} \mathrm{C}\right)$. The group in the presence of FITC-CHSP NPs but without inhibitor treatment was used as control, and their uptake was expressed as $100 \% * P<0.05$, compared with the control value.

Abbreviations: FITC-CHSP, fluorescein isothiocyanate-labeled cholesterolmodified pullulan; HepG2, hepatocellular carcinoma; NP, nanoparticle. uncoated pits (fluid phase), transfers materials to the lysosomal degradative compartment, while caveolae-mediated endocytosis can result in translocation to the ER, Golgi, or through the cell by trancytosis. ${ }^{46}$ Understanding the intracellular fate of NPs with respect to their uptake pattern is crucial in designing a new macromolecular carrier.

Here, to track the CHSP NPs, Golgi apparatus, ER, and lysosomes were immunofluorescently stained. The position of the nucleus in each cell was labeled with DAPI staining. The intracellular localization of the CHSP NPs was traced at different time points. As shown in Figure 10, the colocalization of NPs with either the Golgi apparatus (shown by Giantin) or the ER (shown by Calnexin) was not observed throughout the entire course of the study. Instead, NPs were found colocalized with lysosomes (shown by LAMP2), at 1 hour after incubation. Interestingly, as the incubation time increased, the NPs entrapped in the lysosomal compartments
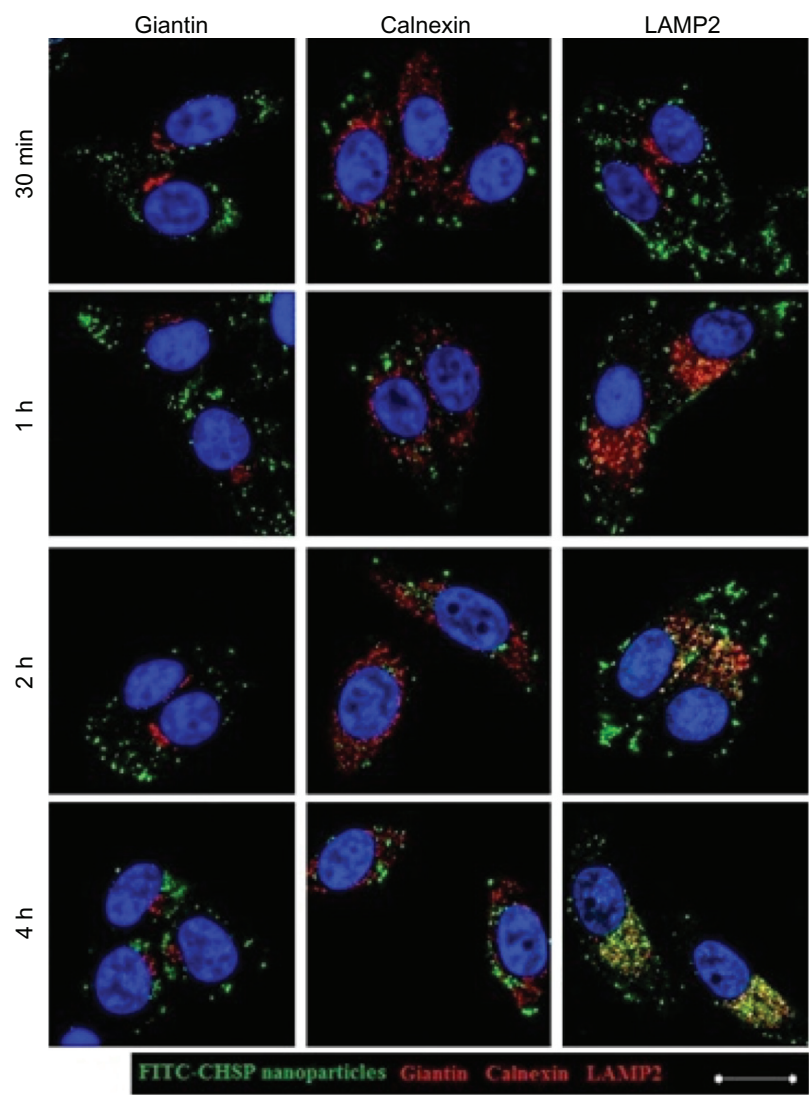

Figure 10 Confocal images of the intracellular trafficking of CHSP NPs at the indicated times using immunofluorescence staining to identify the Golgi apparatus (Giantin), endoplasmic reticula (Calnexin), and lysosomes (LAMP2).

Notes: The nucleus in each cell was labeled with DAPI staining (blue color). Colocalization of NPs (green color) with either the Golgi apparatus (Giantin, red color) or the ER (Calnexin, red color) was not observed. Instead, NPs were found colocalized with lysosomes (LAMP2, yellow color in merged images) at I hour. Scale bar $=20 \mu \mathrm{m}$.

Abbreviations: CHSP, cholesterol-modified pullulan, LAMP2, lysosomal-associated membrane protein 2; NP, nanoparticle. 
gradually migrated toward the perinuclear region. A similar study showed the time-dependent movement of HeLa lysosomes containing hydrophobically modified glycol chitosan NPs toward the nucleus. ${ }^{26}$

\section{Conclusion}

In conclusion, the cellular uptake of CHSP NPs was concentration-, time-, and temperature-dependent. Pretreatment of HepG2 cells with several endocytic inhibitors (eg, chlorpromazine, filipin, and amiloride) indicated that more than one mechanism was involved simultaneously in the NP uptake. Moreover, colocalization studies showed that some of the CHSP NPs were trapped in the lysosomal compartment, while colocalization of NPs with either the Golgi apparatus or the ER was not observed during the entire course of the study. In summary, our findings suggest that the CHSP NPs have potential for improving drug delivery in the treatment of lysosomal storage diseases, cancer, and Alzheimer's disease.

\section{Acknowledgment}

This work was supported by the Doctoral Fund of the Ministry of Education of The People's Republic of China (Grant No 20101106110042).

\section{Disclosure}

The authors declare no conflicts of interest in this work.

\section{References}

1. Singh RS, Saini GK, Kennedy JF. Pullulan: Microbial sources, production and applications. Carbohyd Polym. 2008;73(4):515-531.

2. Rekha MR, Sharma CP. Pullulan as a promising biomaterial for biomedical applications: a perspective. Trends Biomater Artif Organs. 2007;20(2):116-121.

3. Leathers TD. Biotechnological production and applications of pullulan. Appl Microbiol Biotechnol. 2003;62(5-6):468-473.

4. Shingel KI. Current knowledge on biosynthesis, biological activity, and chemical modification of the exopolysaccharide, pullulan. Carbohydr Res. 2004;339(3):447-460.

5. Liu Z, Jiao Y, Wang Y, Zhou C, Zhang Z. Polysaccharides-based nanoparticles as drug delivery systems. Adv Drug Deliver Rev. 2008;60(15): $1650-1662$.

6. Jeong YI, Na HS, Oh JS, Choi KC, Song CE, Lee HC. Adriamycin release from self-assembling nanospheres of poly(DL-lactide-coglycolide)-grafted pullulan. Int J Pharm. 2006;322(1-2):154-160.

7. Zhang HZ, Gao FP, Liu LR, et al. Pullulan acetate nanoparticles prepared by solvent diffusion method for epirubicin chemotherapy. Colloids Surf B Interfaces. 2009;71(1):19-26.

8. Jung SW, Jeong YI, Kim SH. Characterization of hydrophobized pullulan with various hydrophobicities. Int J Pharm. 2003;254(2):109-121.

9. Zhang HZ, Li XM, Gao FP, Liu LR, Zhou ZM, Zhang QQ. Preparation of folate-modified pullulan acetate nanoparticles for tumor-targeted drug delivery. Drug Deliv. 2010;17(1):48-57.

10. Na K, Lee KH, Bae YH. pH-sensitivity and $\mathrm{pH}$-dependent interior structural change of self-assembled hydrogel nanoparticles of pullulan acetate/oligo-sulfonamide conjugate. J Control Release. 2004;97(3): $513-525$.
11. Huang L, Chen $\mathrm{H}$, Zheng Y, et al. Nanoformulation of D- $\alpha$-tocopheryl polyethylene glycol 1000 succinate-b-poly( $\varepsilon$-caprolactoneran-glycolide) diblock copolymer for breast cancer therapy. Integr Biol (Camb). 2011;3(10):993-1002.

12. Na K, Burn Lee T, Park KH, Shin EK, Lee YB, Choi HK. Selfassembled nanoparticles of hydrophobically-modified polysaccharide bearing vitamin $\mathrm{H}$ as a targeted anti-cancer drug delivery system. Eur J Pharm Sci. 2003;18(2):165-173.

13. Akiyoshi K, Deguchi S, Moriguchi N, Yamaguchi S, Sunamoto J. Self-aggregates of hydrophobized polysaccharides in water. Formation and characteristics of nanoparticles. Macromolecules. 1993;26(12): 3062-3068.

14. Akiyoshi K, Deguchi S, Tajima T, Nishikawa T, Sunamoto J. Microscopic structure and thermoresponsiveness of a hydrogel nanoparticle by self-assembly of a hydrophobized polysaccharide. Macromolecules. 1997;30(4):857-861.

15. Nishikawa T, Akiyoshi K, Sunamoto J. Supramolecular assembly between nanoparticles of hydrophobized polysaccharide and soluble protein complexation between the self-aggregate of cholesterolbearing pullulan and a-chymotrypsin. Macromolecules. 1994;27(26): 7654-7659.

16. Nishikawa T, Akiyoshi K, Sunamoto J. Macromolecular complexation between bovine serum albumin and the self-assembled hydrogel nanoparticle of hydrophobized polysaccharides. J Am Chem Soc. 1996;118(26):6110-6115.

17. Akiyoshi K, Kobayashi S, Shichibe S, et al. Self-assembled hydrogel nanoparticle of cholesterol-bearing pullulan as a carrier of protein drugs: complexation and stabilization of insulin. $J$ Control Release. 1998;54(3):313-320.

18. Akiyoshi K, Sasaki Y, Kuroda K, Sunamoto J. Controlled association of hydrophobized polysaccharide by cyclodextrin. Chem Lett. 1998;27(1):93-94

19. Taniguchi I, Akiyoshi K, Sunamoto J, Suda Y, Yamamoto M. Cell specificity of macromolecular assembly of cholesteryl and galactoside groupsconjugated pullulan. J Bioact Compat Polym. 1999;14(3):195-212.

20. Mei L, Zhang Z, Zhao L, et al. Pharmaceutical nanotechnology for oral delivery of anticancer drugs. Adv Drug Deliv Rev. Epub December 7, 2012.

21. Nomura Y, Ikeda M, Yamaguchi N, Aoyama Y, Akiyoshi K. Protein refolding assisted by self-assembled nanogels as novel artificial molecular chaperone. FEBS Lett. 2003;553(3):271-276.

22. Hirakura T, Nomura Y, Aoyama Y, Akiyoshi K. Photoresponsive nanogels formed by the self-assembly of spiropyrane-bearing pullulan that act as artificial molecular chaperones. Biomacromolecules. 2004;5(5):1804-1809.

23. Nomura Y, Sasaki Y, Takagi M, Narita T, Aoyama Y, Akiyoshi K. Thermoresposive controlled association of protein with a dynamic nanogel of hydrophobized polysaccharide and cyclodextrin: heat shock protein-like activity of artificial molecular chaperone. Biomacromolecules. 2005;6(1):447-452.

24. Yang WZ, Chen HL, Gao FP, et al. Self-aggregated nanoparticles of cholesterol-modified pullulan conjugate as a novel carrier of mitoxantronep. Curr Nanosci. 2010;6(3):298-306.

25. Chiu YL, Ho YC, Chen YM, et al. The characteristics, cellular uptake and intracellular trafficking of nanoparticles made of hydrophobicallymodified chitosan. J Control Release. 2010;146(1):152-159.

26. Nam HY, Kwon SM, Chung H, et al. Cellular uptake mechanism and intracellular fate of hydrophobically modified glycol chitosan nanoparticles. J Control Release. 2009;135(3):259-267.

27. Delie F. Evaluation of nano- and microparticle uptake by the gastrointestinal tract. Adv Drug Deliver Rev. 1998;34(2-3):221-223.

28. Zhang Y, Tang L, Sun L, et al. A novel paclitaxel-loaded poly(epsiloncaprolactone)/Poloxamer 188 blend nanoparticle overcoming multidrug resistance for cancer treatment. Acta Biomater. 2010;6(6):2045-2052.

29. Ma Y, Zheng Y, Zeng X, et al. Novel docetaxel-loaded nanoparticles based on PCL-Tween 80 copolymer for cancer treatment. Int $J$ Nanomedicine. 2011;6:2679-2688. 
30. Kaneo Y, Tanaka T, Nakano T, Yamaguchi Y. Evidence for receptormediated hepatic uptake of pullulan in rats. J Control Release. 2001; 70(3):365-373.

31. Mosmann T. Rapid colorimetric assay for cellular growth and survival: application to proliferation and cytotoxicity assays. J Immunol Methods. 1983;65(1-2):55-63.

32. Perumal OP, Inapagolla R, Kannan S, Kannan RM. The effect of surface functionality on cellular trafficking of dendrimers. Biomaterials. 2008;29(24-25):3469-3476.

33. Rekha MR, Sharma CP. Blood compatibility and in vitro transfection studies on cationically modified pullulan for liver cell targeted gene delivery. Biomaterials. 2009;30(34):6655-6664.

34. Huang M, Ma Z, Khor E, Lim LY. Uptake of FITC-chitosan nanoparticles by A549 cells. Pharm Res. 2002;19(10):1488-1494.

35. Suh H, Jeong B, Liu F, Kim SW. Cellular uptake study of biodegradable nanoparticles in vascular smooth muscle cells. Pharm Res. 1998; 15(9):1495-1498.

36. Rekha MR, Sharma CP. Hemocompatible pullulan-polyethyleneimine conjugates for liver cell gene delivery: In vitro evaluation of cellular uptake, intracellular trafficking and transfection efficiency. Acta Biomater. 2011;7(1):370-379.

37. Choi Y, Thomas T, Kotlyar A, Islam MT, Baker JR Jr. Synthesis and functional evaluation of DNA-assembled polyamidoamine dendrimer clusters for cancer cell-specific targeting. Chem Biol. 2005;12(1): $35-43$.
38. Rejman J, Bragonzi A, Conese M. Role of clathrin-and caveolaemediated endocytosis in gene transfer mediated by lipo- and polyplexes. Mol Ther. 2005;12(3):468-474.

39. Lai SK, Hida K, Man ST, et al. Privileged delivery of polymer nanoparticles to the perinuclear region of live cells via a non-clathrin, nondegradative pathway. Biomaterials. 2007;28(18):2876-2884.

40. Parton RG, Simons K. The multiple faces of caveolae. Nat Rev Mol Cell Biol. 2007;8(3):185-194.

41. Mayor S, Pagano RE. Pathways of clathrin-independent endocytosis. Nat Rev Mol Cell Biol. 2007;8(8):603-612.

42. Orlandi PA, Fishman PH. Filipin-dependent inhibition of cholera toxin: evidence for toxin internalization and activation through caveolae-like domains. J Cell Biol. 1998;141(4):905-915.

43. Fujimoto T, Kogo H, Nomura R, Une T. Isoforms of caveolin-1 and caveolar structure. J Cell Sci. 2000;113 Pt 19:3509-3517.

44. Hewlett LJ, Prescott AR, Watts C. The coated pit and macropinocytic pathways serve distinct endosome populations. J Cell Biol. 1994;124(5): 689-703.

45. Jones AT, Gumbleton M, Duncan R. Understanding endocytic pathways and intracellular trafficking. A prerequisite for effective design of advanced drug delivery systems. Adv Drug Deliv Rev. 2003;55(11): 1353-1357.

46. Moore MN. Do nanoparticles present ecotoxicological risks for the health of the aquatic environment? Environ Int. 2006;32(8): 967-976.
International Journal of Nanomedicine

\section{Publish your work in this journal}

The International Journal of Nanomedicine is an international, peerreviewed journal focusing on the application of nanotechnology in diagnostics, therapeutics, and drug delivery systems throughout the biomedical field. This journal is indexed on PubMed Central, MedLine, CAS, SciSearch $\AA$, Current Contents ${ }^{\circledR} /$ Clinical Medicine,

\section{Dovepress}

Journal Citation Reports/Science Edition, EMBase, Scopus and the Elsevier Bibliographic databases. The manuscript management system is completely online and includes a very quick and fair peer-review system, which is all easy to use. Visit http://www.dovepress.com/ testimonials.php to read real quotes from published authors. 